\title{
Auswirkungen der Klimaänderung auf Österreichs Wasserwirtschaft - ein aktualisierter Statusbericht
}

\author{
Günter Blöschl · Alfred Paul Blaschke · Klaus Haslinger · Michael Hofstätter • Juraj Parajka · José Salinas • \\ Wolfgang Schöner
}

Online publiziert: 2. Juli 2018

(c) Der/die Autor(en) 2018

Zusammenfassung Dieser Beitrag fasst die Ergebnisse der Studie „Klimawandel in der Wasserwirtschaft - Schwerpunkt Hochwasser und Dürre" zusammen, die den aktuellen Stand des Wissens der Klimaänderungen in Hinblick auf die Auswirkungen auf die österreichische Wasserwirtschaft darstellt. Die Verdunstung hat in den letzten drei Jahrzehnten, genauso wie der Jahresniederschlag, im Mittel für Österreich um etwa $80 \mathrm{~mm} /$ a zugenommen. Die zunehmenden Trends der gemessenen Hochwasserabflüsse haben sich in den letzten Jahren etwas verstärkt, die abnehmenden Trends der gemessenen Niederwasserabflüsse etwas abgeschwächt. Klimaprojektionen lassen gewisse Zunahmen der Hochwasser erwarten, die jedoch wesentlich kleiner sind als ihre natürlichen Schwankungen. In den Alpen werden deutlich höhere Abflüsse im Winter erwartet, in den Flachlandregionen Ost- und Südösterreichs eher eine Abnahme der Abflüsse bei Niederwasser. Insgesamt sind die Änderungen im Vergleich zur Vorgängerstudie aus dem Jahr 2011 nicht

Univ.-Prof. DI Dr. G. Blöschl (凶) . Univ.-Prof. DI Dr. A. P. Blaschke . PD J. Parajka, PhD · Ing. Dr. J. Salinas Institut für Wasserbau und Ingenieurhydrologie, Technische Universität Wien, Karlsplatz 13, 1040 Wien, Österreich bloeschl@hydro.tuwien.ac.at

Mag. K. Haslinger · Mag. M. Hofstätter Zentralanstalt für Meteorologie und Geodynamik (ZAMG), Hohe Warte 38, 1190 Wien, Österreich

Univ.-Prof. Dr. W. Schöner Institut für Geographie und Raumforschung, Karl-Franzens-Universität Graz Heinrichstraße 36, 8010 Graz, Österreich sehr groß. Einige zusätzliche Empfehlungen werden gegeben.

\section{Impact of climate change on \\ Austria's water sector-an updated status report}

Abstract This paper summarises the results of the study "Climate change and water management - a focus on floods and droughts" which presents the current state of knowledge of climate change with regard to the effects on water management in Austria. Average evaporation in Austria has increased by about $80 \mathrm{~mm} / \mathrm{yr}$ over the past three decades, similar to annual rainfall. The increasing trends in measured flood discharges have become somewhat more frequent in recent years, while the decreasing trends of measured low flows have weakened to some extent. Climate projections suggest some increases in floods, but these are much smaller than the natural flood variability. In the Alps, significantly higher winter runoff is expected. In the lowland regions of eastern and southern Austria, low flows will possibly decrease. Overall, the findings are similar to those of the previous study from the year 2011. Some additional recommendations are given.

\section{Einleitung}

Seit der letzten Bewertung möglicher Anpassungsstrategien an den Klimawandel für Österreichs Wasserwirtschaft im Jahr 2011 (ZAMG und TU Wien 2011; Blöschl et al. 2011a) sind eine Reihe von zusätzlichen Informationen verfügbar geworden. Zum einen liegen nun die Ergebnisse mehrerer neuer Projekte zu dem Thema vor. Zum anderen sind sieben zusätzliche Jahre an hydrologischen Daten verfügbar, in denen große Hochwasserereignisse und Trockenperioden in Österreich aufgetreten sind. Es stellt sich nun die
Frage, ob die im Jahr 2011 getroffene Einschätzung weiterhin gültig ist. Das damalige Bundesministerium für Land- und Forstwirtschaft, Umwelt und Wasserwirtschaft und die Wasserwirtschaftlichen Abteilungen der Länder beauftragten deshalb eine Studie mit dem Ziel, den aktualisierten Stand des Wissens über Klimaänderung in Österreich in Hinblick auf die Auswirkungen auf die Wasserwirtschaft zu beschreiben (Blöschl et al. 2017a).

Die vorliegende Arbeit fasst die wichtigsten Ergebnisse dieser Studie zusammen. Betrachtet werden die Schwerpunkte Hochwasser und Dürre. Wie die Vorgängerstudie (ZAMG und TU Wien 2011; Blöschl et al. 2011a) widmet sich diese Studie der Beurteilung der Einflüsse des Klimawandels auf die Wasserwirtschaft in Österreich. Andere, insbesondere direkte anthropogene Effekte wurden zur besseren Fokussierung ausgeklammert. Manchmal sind jedoch klimainduzierte und anthropogene Effekte nicht leicht zu trennen. Der Klimawandel wird für die Wasserwirtschaft und für den „natürlichen Zustand“ der Gewässer nach EU Wasserrahmenrichtlinie als eine „natürliche" Veränderung und nicht als anthropogen verursachte Veränderung der Umweltbedingungen betrachtet, da der Einfluss nationaler wasserwirtschaftlicher Maßnahmen auf das Klima vernachlässigbar gering ist (Montanari et al. 2010). Die globalen Einflüsse des Menschen auf das Klima werden in dieser Studie in Form von Szenarien berücksichtigt. Die Auswertungen erfolgen räumlich differenziert für das gesamte Bundesgebiet. Es handelt sich also um regionale Untersuchungen, die Detailstudien nicht ersetzen können.

Wegen der großen Unsicherheiten bei der Einschätzung der Einflüsse des Klimawandels auf die Wasserwirtschaft verfolgte die Studie drei Grundprinzipien: (1) Weiche und harte Aussagen wurden im Sinne der Sicherheit der 


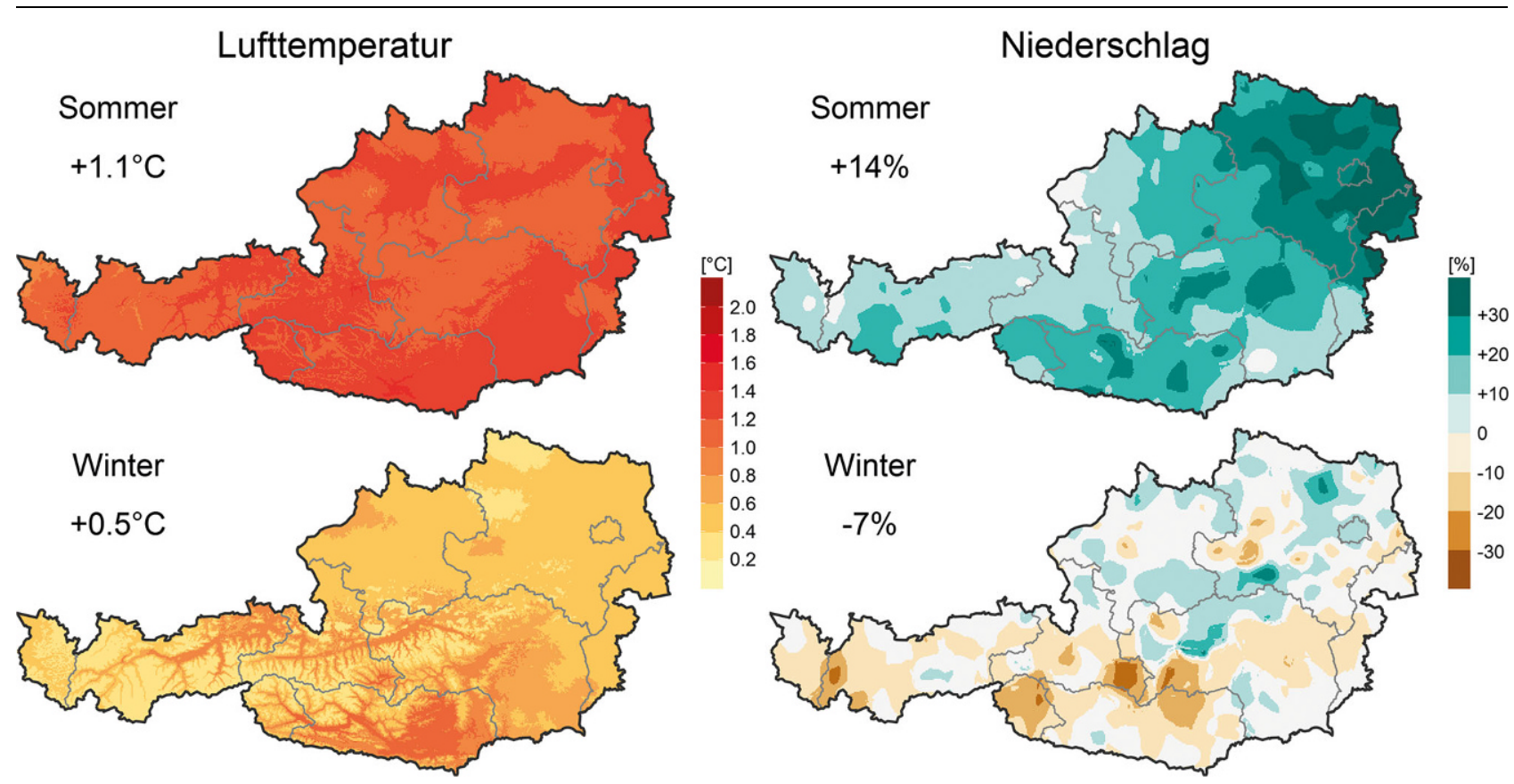

Abb. 1 Beobachtete Änderung der mittleren Lufttemperatur und des Niederschlags im Sommer (Juni-August) und Winter (Dezember-Februar) für den Zeitraum 1996-2014 im Vergleich zum Zeitraum 1976-1995 (Hiebl und Frei 2016)

Aussagekraft unterschieden; (2) Einander ergänzende Informationsquellen wurden herangezogen, um die Zuverlässigkeit der Aussage zu erhöhen und einzuschätzen; (3) Der Fokus wurde auf Mechanismen gelegt, um differenziertere und transparentere Aussagen über Ursache-Wirkung als durch Szenarien alleine zu erhalten. Die Beurteilung erfolgt getrennt für die Situation in der Vergangenheit und die Situation in der Zukunft.

\section{Klimavariabilität und Wasserdargebot}

\subsection{Situation in der Vergangenheit}

Der Alpenraum war bis jetzt stärker vom globalen Klimawandel betroffen als andere Gebiete der Erde. Seit dem Jahr 1970 stieg in Österreich die Temperatur um $+2^{\circ}$ an, während die Nordhemisphäre lediglich einen Anstieg von $+1,5^{\circ}$ aufweist. Abb. 1 zeigt die jahreszeitliche Änderung der Mitteltemperatur in Österreich für den Zeitraum 1996-2014 im Vergleich zum Zeitraum 1976-1995. Generell zeigt der Sommer eine größere Temperaturänderung als der Winter. Die letzten Jahre waren besonders warm, sodass die aktualisierte Periode in Abb. 1 eine etwas größere Temperaturänderung zur Referenzperiode zeigt als die Vorgängerstudie $\left(+1,2^{\circ} \mathrm{C}\right.$ Jahresmittel, Vorgängerstudie $+1,0^{\circ} \mathrm{C}$ für $1996-2007 \mathrm{im}$ Vergleich zu 1976-1995). Der Sommerniederschlag nahm im Vergleich zur Referenzperiode im Mittel über Österreich um $+14 \%$ $\mathrm{zu}$ (Vorgängerstudie $+12 \%$ ). Im Nordosten Österreichs war die Zunahme bis $\mathrm{zu} 40 \%$. Der Winterniederschlag nahm im Mittel über Österreich um $-7 \%$ ab (Vorgängerstudie $-12 \%$ ), wobei die Abnahme im Süden Österreichs am größten ist. In der Periode der Vorgängerstudie war die Abnahme im Süden allerdings noch wesentlich stärker (bis $\mathrm{zu}-40 \%$ ) (harte Aussagen).

Neue Informationen liegen auch für die Veränderungen des Schnees in Österreich vor. Sowohl die Schneehöhe als auch die Neuschneesumme haben seit 1961 stark abgenommen, besonders im Süden und Westen Österreichs (Abb. 2) (harte Aussagen). Die kleinen Veränderungen im Nordosten Österreich sind etwas überraschend und spiegeln die Komplexität der alpinen Schneeklimatologie wider, insbesondere die komplexe Interaktion der Schneefallgrenze mit der Orografie (siehe z.B. Minder 2010). Aus den Ergebnissen der Trenduntersuchung kann geschlossen werden, dass eine allgemeine Zunahme der Lufttemperatur nicht gleichbedeutend mit abnehmenden Neuschneemengen und Schneehöhen ist. Es sind auch die Veränderungen des Niederschlags zu berücksichtigen. Schöner et al. (2016) zeigten, dass in tiefen Lagen die mittlere Schneehöhe im Winter (Dezember bis Februar) sensitiv auf Änderungen der Lufttemperatur reagiert, während sie in hohen Lagen sensitiv auf Änderungen des Niederschlags reagiert. Dies hängt mit der Grenztemperatur von ca. $0^{\circ} \mathrm{C}$ zusammen, die zwischen festem und flüssigem Niederschlag entscheidet.

Zur Beurteilung der aktualisierten Situation der Wasserbilanz zeigt Abb. 3 die mittlere Wasserbilanz von 166 Pegeleinzugsgebieten in Österreich für den Zeitraum 1976-2014. Der Jahresniederschlag im Zeitraum 1995-2014 war auf einem relativ hohen Niveau im Vergleich zum Mittelwert des 20. Jahrhunderts. Im Zeitraum 1995-2014 war der mittlere Niederschlag in Österreich etwa $80 \mathrm{~mm} / \mathrm{a}$ höher als im Zeitraum 1976-1990. Demgegenüber hat sich der Jahresabfluss im Zeitraum 1976-2014 im Mittel für Österreich wenig geändert. Die Differenz aus Niederschlag und Abfluss (Verdunstung plus Speicheränderung) muss dementsprechend um etwa $80 \mathrm{~mm} / \mathrm{a}$ zugenommen haben. Da anzunehmen ist, dass die Speicheränderung über einen Zeitraum von Dekaden relativ klein ist im Vergleich zu den anderen Komponenten der Wasserbilanz, kann abgeschätzt werden, dass die Verdunstung im Mittel für Österreich um etwa $80 \mathrm{~mm} / \mathrm{a}$ zugenommen 


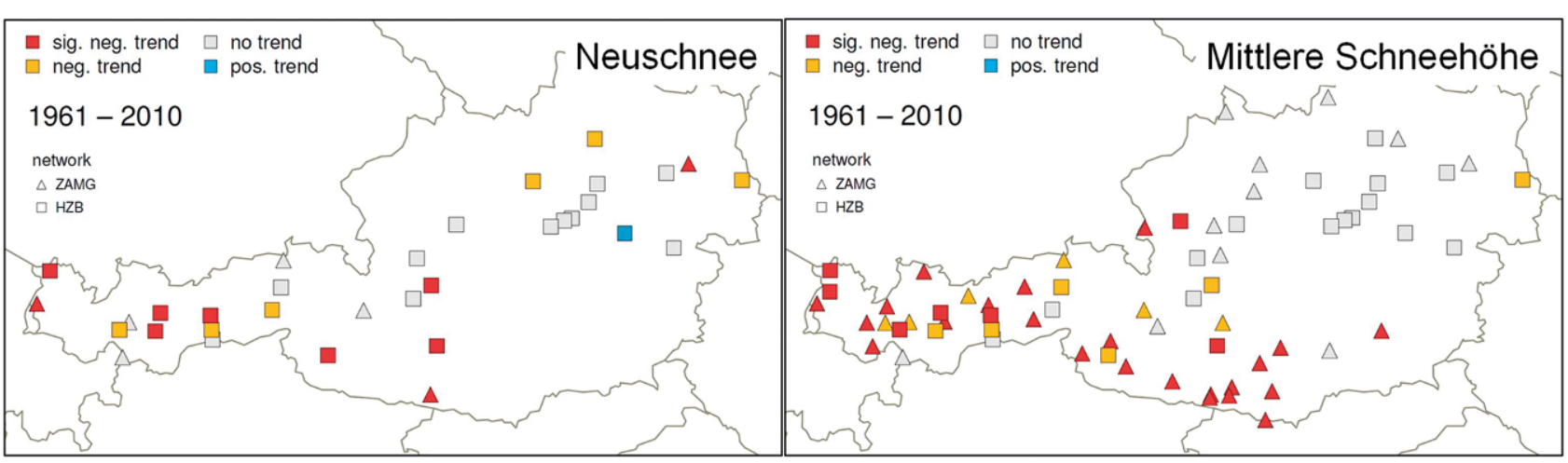

Abb. 2 Trends der täglichen Neuschneesumme (links) und der Gesamtschneehöhe (rechts) gemittelt über die Periode November-April jeden Jahres für den Zeitraum 1961-2010. Orange: signifikant negativ (5\% Signifikanzniveau); Rot: signifikant negativ (1 \% Signifikanzniveau); Grau: Trends nicht signifikant; Blau: signifikant positiv (5\% Signifikanzniveau) (Schöner et al. 2016)
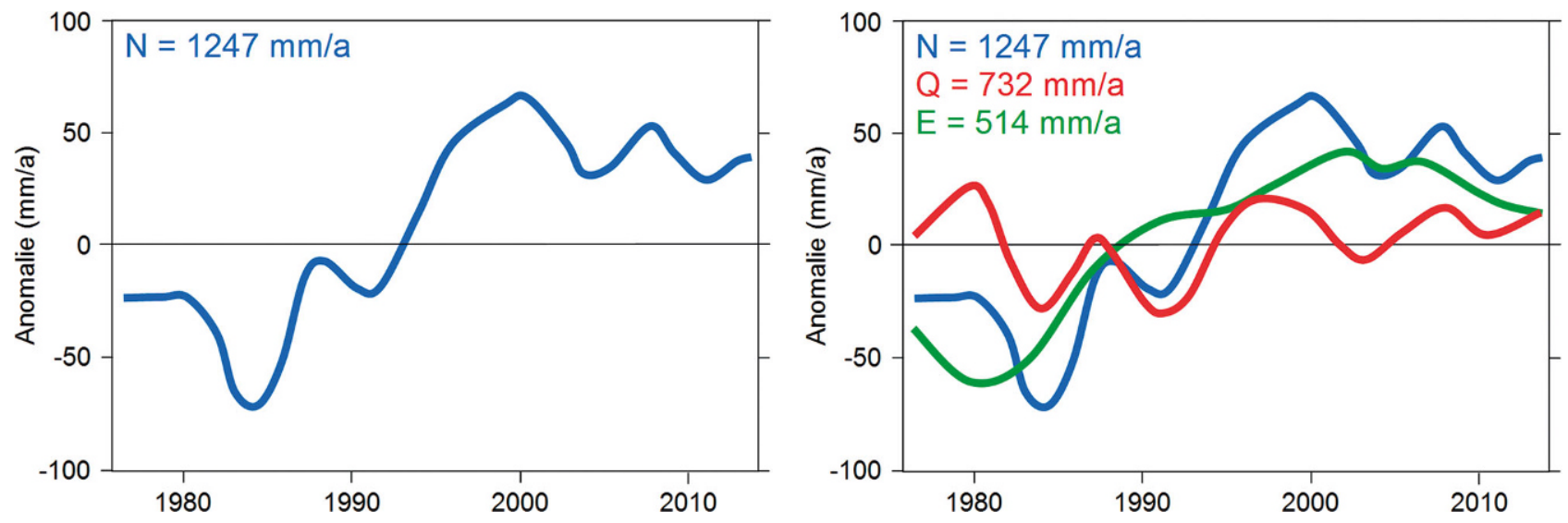

Abb. 3 Anomalien des mittleren Jahresniederschlags (blau) im Vergleich zum Mittelwert 1976-2014 von 166 Pegeleinzugsgebieten in Österreich. Abfluss (rot). Differenz von Niederschlag und Abfluss (grün). Gleitendes Mittel über 20 Jahre (Duethmann und Blöschl 2018)

hat (harte Aussage). Die Zunahme der Verdunstung ist zu $38 \%$ auf die Zunahme der Lufttemperatur und der Strahlung zurückzuführen, zu $30 \%$ auf die längere Vegetationsperiode und zu $32 \%$ auf die erhöhte Bodenfeuchte (Duethmann und Blöschl 2018) (mittelharte Aussage).

\subsection{Situation in der Zukunft}

Für Europa liegen neue regionale Klimamodellsimulationen vor (EUROCORDEX, Giorgi et al. 2016). Diese zeigen auch weiterhin ein räumlich stark differenziertes Bild der zukünftigen Erwärmung mit dem geringsten Temperaturanstieg über Mittel- und Westeuropa und dem stärksten über Nordeuropa und dem Westen Russlands (harte Aussage). Weiterhin ist mit einer Zunahme des Niederschlags über Skandinavien und einer Abnahme im Mittelmeerraum zu rechnen (mittelharte Aussage). Die neuen hoch aufgelösten ÖKS15-
Simulationen zeigen im Mittel für Österreich für 2021-2050 im Vergleich zu 1971-2000 eine Erwärmung von $+1,5^{\circ} \mathrm{C}$. Diese Erwärmung weist keine nennenswerten saisonalen Unterschiede auf (harte Aussage). Der Winterniederschlag nimmt gemäß den Simulationen um $+14 \% \mathrm{zu}$, der Sommerniederschlag nur um $+3 \%$, der Jahresniederschlag um $6 \%$ (mittelharte Aussage). Die Änderungen sind räumlich sehr ähnlich, nur im Winter dürfte die Zunahme im Nordosten etwas stärker sein als auf der Alpensüdseite (weiche Aussage) (Abb. 4). Zeigte die Vorgängerstudie noch eine Abnahme des Winterniederschlags südlich der Alpen von 0 bis $10 \%$, so ergeben die neuen Simulationen eine Zunahme von etwa +10 bis $+15 \%$. Dieses Signal wird jedoch erheblich durch die relativ trockene Referenzperiode von 1971-2000 (Alpensüdseite) bestimmt und darf nicht als anthropogenes Klimasignal interpretiert werden. Trotz der mittlerweile hohen räumlichen Auflö- sung der regionalen Klimamodelle werden bestimmte Niederschlagsprozesse noch immer nicht explizit erfasst, wie beispielsweise der konvektive Anteil des Niederschlags. Aus diesem Grund muss auch weiterhin vor allem der sommerliche Niederschlag als unsicher eingestuft werden.

Die Bewertung der Wasserbilanz der Vorgängerstudie bleibt im Wesentlichen bestehen. Die natürliche Variabilität des mittleren jährlichen Abflusses zwischen den Jahren ist größer als die zufolge Klimaänderung im Zeitraum 2021-2050 $\mathrm{zu}$ erwartenden Änderungen (mittelharte Aussage). Geht man davon aus, dass auch in Zukunft die Temperatur in ähnlicher Weise ansteigen wird, und nimmt man gleich bleibenden Niederschlag an, so ist damit $\mathrm{zu}$ rechnen, dass die Gebiete trockener werden. Im Südosten ist möglicherweise eine Abnahme des mittleren jährlichen Abflusses 2021-2050 im Vergleich zu 1976-2006 zu erwarten (um ca. 5\%) 


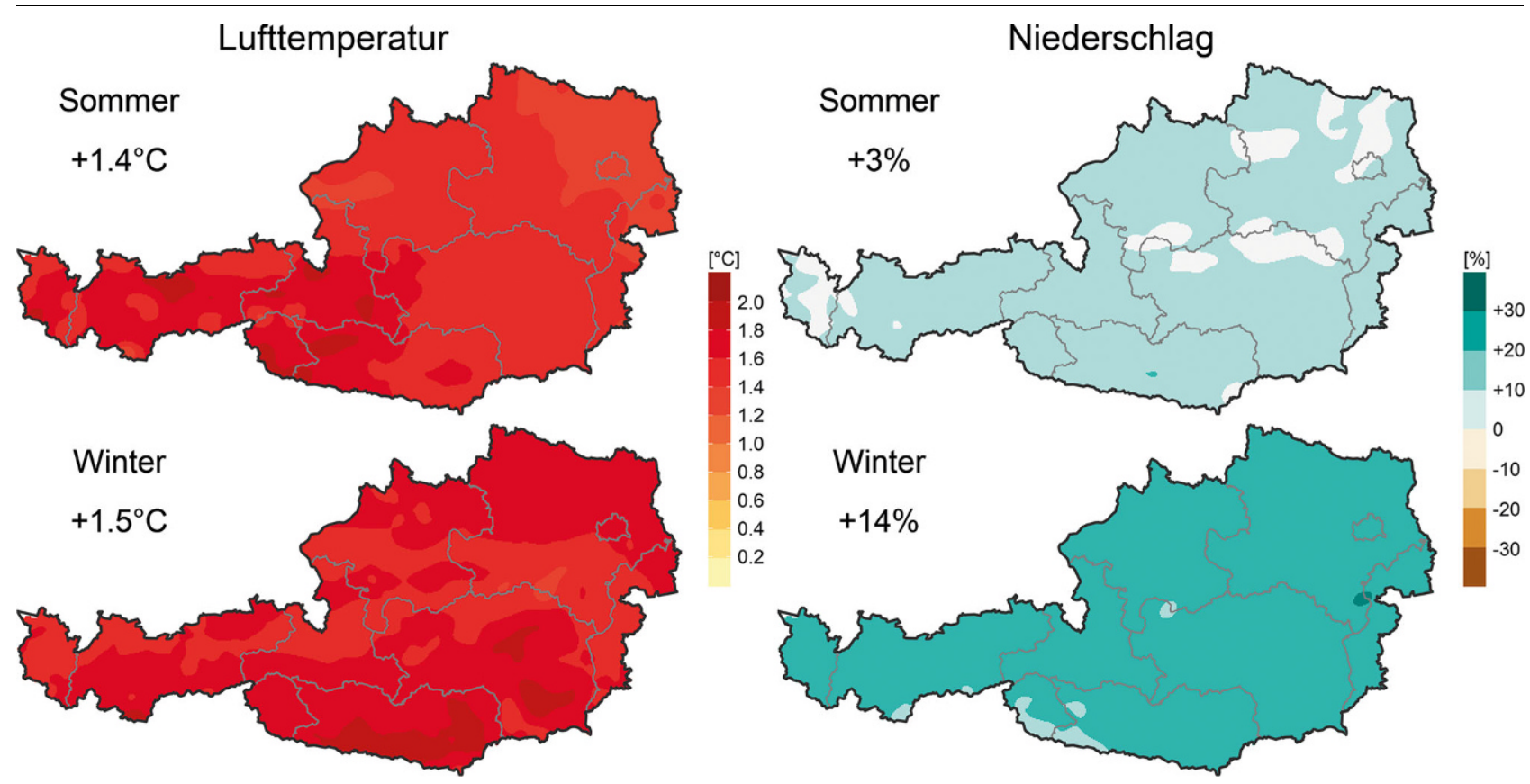

Abb. 4 Projizierte Änderung der mittleren Lufttemperatur und des Niederschlags im Sommer (Juni-August) und Winter (Dezember-Februar) für den Zeitraum2021-2050 im Vergleich zu 1971-2000. RegionaleSimulationenÖKS15 basierend aufEURO-CORDEX für das Szenario RCP8.5 (Chimani et al. 2016)

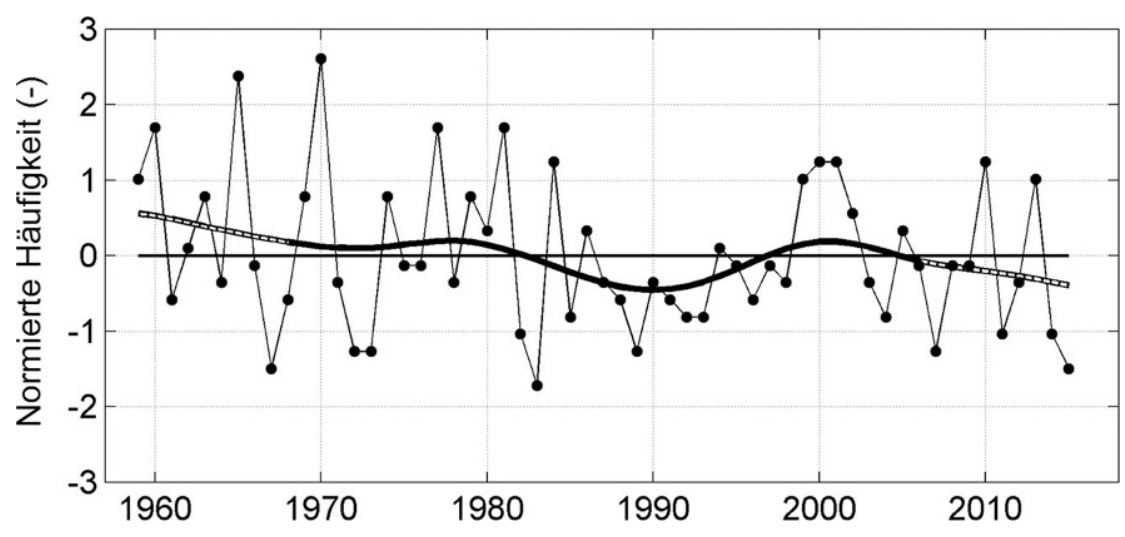

Abb. 5 Jährliche Häufigkeit der Zugbahn Vbals Kombination der Zugbahnanalyse auf Bodenluftdruck- und dem 700-hPa-Niveau basierend auf den japanischen JRAReanalysen (Hofstätter et al. 2015)

(weiche Aussage). In ganz Österreich ist eine Erhöhung der Winterabflüsse zu erwarten. In Norden dürfte sie etwas kleiner ausfallen als in der Vorgängerstudie angegeben (10-20\%), im Süden etwas größer (5-15\%) (mittelharte Aussage). Im Osten (Flachland) ist eine Abnahme des Abflusses im Sommer und Frühjahr, und im Westen (Alpen) eine Abnahme des Abflusses im Sommer um 0-10\% zu erwarten (weiche Aussage). Viglione et al. 2016; Alaoui et al. 2018;

\section{Hochwasser}

\subsection{Situation in der Vergangenheit}

Hochwässer können im Wesentlichen durch drei Faktoren verstärkt werden: Klima, Landnutzung und wasserbauliche Maßnahmen (Hall et al. 2014; Blöschl et al. 2018). Hinweise auf Änderungen von hochwasserrelevanten Faktoren im Klimasystem in Mitteleuropa liegen vor (Hofstätter et al. 2015, 2017; Blöschl et al. 2017b). Die Häufigkeit von starkniederschlagsrelevanten Zugbahnen oder Wetterlagen im Zeitraum
1959-2015 hat jedoch nicht zugenommen, auch nicht für den in Mitteleuropa besonders relevanten Zugbahntyp $\mathrm{Vb}$ (Abb. 5). In der Periode 1986-2010 hat in Österreich die Anzahl von Tagen mit großen 24-stündlichen Niederschlagsummen ( $>90$. Perzentil) um $+10 \%$ bis $+15 \%$ zugenommen und gleichzeitig die Anzahl der schwachen $(<50$. Perzentil) abgenommen (mittelharte Aussage) (Chimani et al. 2016). Wie das Hochwasser im Juni 2013 an der Donau und Zubringern gezeigt hat, wird die Größe von Hochwässern nicht nur durch den Ereignisniederschlag, sondern durch die Kombination von Niederschlag mit den Gebietsbedingungen (Bodenfeuchte, Schnee) bestimmt (Blöschl et al. 2013). Hauptverantwortlich für die außergewöhnlich großen Abflüsse des Hochwassers im Juni 2013 waren die hohen Niederschläge am Alpennordrand kombiniert mit einer hohen Bodenfeuchte zu Beginn des Ereignisses. Auswertungen stündlicher Niederschlagsdaten für den Zeitraum 1986-2015 zeigten keine graduelle und signifikante Veränderung konvektiver Starkniederschläge, sondern zwei Phasen (1989-1994 und 2006-2014) hoher konvektiver Aktivität (mittelharte Aussage). Während für Tagesniederschläge gezeigt wurde, dass der Niederschlag mit der Lufttemperatur zunimmt, konnte dies für kürzere (konvektive) Niederschläge nicht ge- 


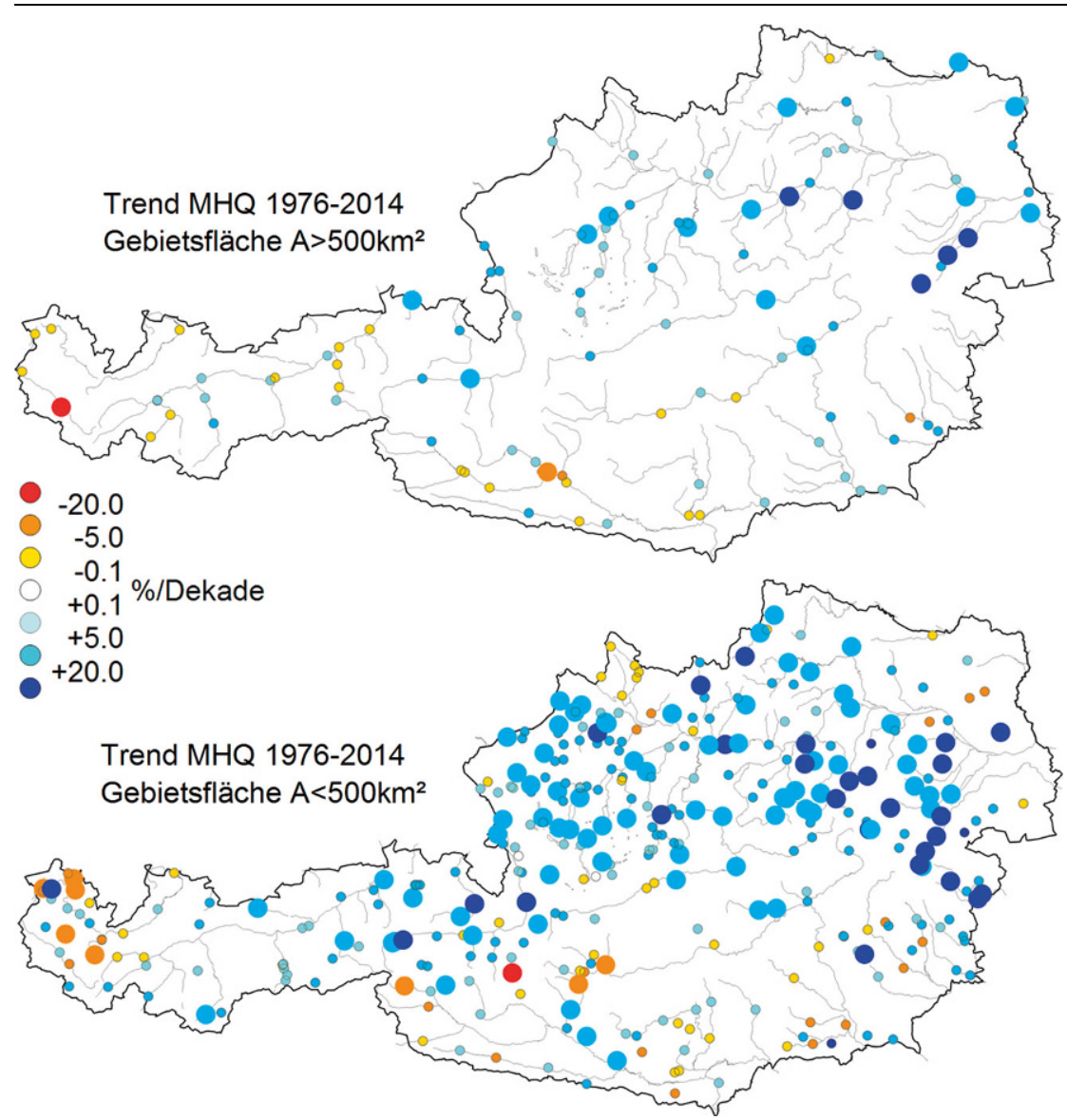

Abb. 6 Trends der Jahresmaxima der Hochwässerfür denZeitraum 1976-2014.Große Kreise blau: signifikant steigende Trends, d. h. Zunahme des mittleren jährlichen Hochwassers MHQ. Große Kreise rot: signifikant fallende Trends. Kleine Kreise:Trends nicht signifikant (Signifikanzniveau $5 \%$ ). Oben: Gebiete $>500 \mathrm{~km}^{2}$, unten: Gebiete $<500 \mathrm{~km}^{2}$ Fläche

zeigt werden (Lexer et al. 2018). Die beobachtete Veränderung von konvektiven Starkniederschlägen bleibt mit großen Unsicherheiten behaftet, besonders bei kurzen Dauerstufen $(<3 \mathrm{~h})$.

Trendauswertungen der beobachteten Jahreshochwasserdurchflüsse in Österreich für den Zeitraum 1976-2014 es $17 \%$ (Vorgängerstudie $8 \%$ ). Betrachtet man alle Pegeleinzugsgebiete (große und kleine), dann zeigen $26 \%$ der Gebiete einen signifikant steigenden Trend (Vorgängerstudie 16\%). Wegen der hohen Hochwasserdurchflüsse im Zeitraum 2008-2014 hat also der Anteil der Gebiete mit signifikant steigenden Trends im Vergleich zur Vorgängerstudie zugenommen, sowohl nördlich als auch südlich des Alpenhauptkammes. Diese Zunahme ist auf eine Zunahme der Sommerhochwässer zurückzuführen. $32 \%$ der Gebiete $<500 \mathrm{~km}^{2}$ zeigen einen signifikant steigenden Trend der Sommerhochwässer (Vorgängerstudie $18 \%)$. Der mittlere Trend der Jahreshochwässer blieb mit +6,8\%/Dekade (Vorgängerstudie $+6,6 \% /$ Dekade) ungefähr gleich. Nördlich des Alpenhauptkammes hat sich der zunehmende mittlere Trend etwas abgeschwächt, südlich des Alpenhauptkammes etwas verstärkt. Letztere Beobachtung stimmt mit den etwas höheren Niederschlägen im Süden Österreichs in den letzten Jahren überein.

\subsection{Situation in der Zukunft}

Auswertungen von Hofstätter et al. (2015) auf Basis von drei globalen Klimamodellen lassen erwarten, dass die Häufigkeit starkniederschlagsrelevanter Zugbahnen und Wetterlagen für den Zeitraum 2071-2100 leicht abnehmen wird (mittelharte Aussage). Gleichzeitig ist mit um $+10 \%$ bis $+25 \%$ (Sommer, Abb. 7) bzw. $+15 \%$ bis $+30 \%$ (Winter) größeren Niederschlägen bei Auftreten eines $\mathrm{Vb}$-Tiefs zu rechnen (mittelharte Aussage). Die Änderungen sind in absoluten Zahlen gemessen im Sommer größer als im Winter, da die typischen Starkniederschlagsmengen bei Vb-Ereignissen im Sommer um ca. $50 \%$ größer sind. EURO-CORDEX-Simulationen

Tab. 1 Prozent der Pegel mit signifikant steigenden und fallenden Trends der Jahreshochwasser in Österreich sowie nördlich und südlich des Alpenhauptkammes. Erster Wert: Gebiete $>500 \mathrm{~km}^{2}$, zweiter Wert: Gebiete $<500 \mathrm{~km}^{2}$ Fläche. Entsprechend dem gewählten Signifikanzniveau von $5 \%$ (jeweils $5 \%$ an den beiden Enden der Verteilung) sind Werte von $5 \%$ und kleiner als zufällig anzusehen. Für den Zeitraum 1976-2007 besitzen insgesamt $16 \%$ der Pegel einen signifikant steigenden Trend, für den Zeitraum 1976-2014 sind es $26 \%$ der Pegel

\begin{tabular}{|c|c|c|c|c|c|c|c|}
\hline & \multirow{3}{*}{$\begin{array}{l}\text { Anzahl der } \\
\text { Pegel }\end{array}$} & \multicolumn{3}{|c|}{$\begin{array}{l}\text { 1976-2007 } \\
\text { (min. 25 Jahre) }\end{array}$} & \multicolumn{3}{|c|}{$\begin{array}{l}\text { 1976-2014 } \\
\text { (min. 32 Jahre) }\end{array}$} \\
\hline & & \multicolumn{3}{|c|}{ Anteil d. Pegel [\%] mit } & \multicolumn{3}{|c|}{ Anteil d. Pegel [\%] mit } \\
\hline & & $\begin{array}{l}\text { Steigendem } \\
\text { Trend }\end{array}$ & $\begin{array}{l}\text { Nicht signif. } \\
\text { Trend }\end{array}$ & $\begin{array}{l}\text { Fallendem } \\
\text { Trend }\end{array}$ & $\begin{array}{l}\text { Steigendem } \\
\text { Trend }\end{array}$ & $\begin{array}{l}\text { Nicht signif. } \\
\text { Trend }\end{array}$ & $\begin{array}{l}\text { Fallendem } \\
\text { Trend }\end{array}$ \\
\hline Alle Gebiete in Österreich & $117 / 381$ & $8 / 18$ & $86 / 80$ & $6 / 2$ & $17 / 29$ & $80 / 68$ & $3 / 3$ \\
\hline Rhein, Donau und Elbegebiete & $82 / 281$ & 9/22 & $89 / 78$ & $2 / 0$ & $24 / 34$ & $74 / 64$ & $2 / 2$ \\
\hline Drau, Mur und Raabgebiete & $35 / 100$ & $6 / 9$ & $80 / 84$ & $14 / 7$ & $3 / 15$ & $94 / 81$ & $3 / 4$ \\
\hline
\end{tabular}




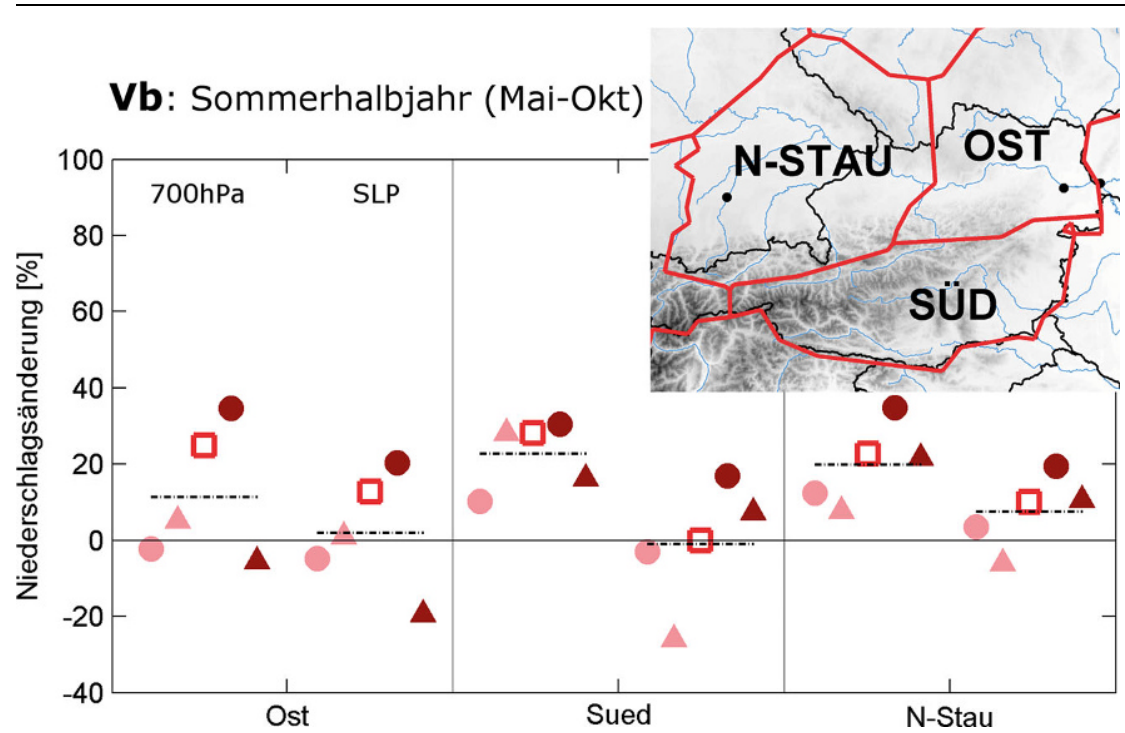

Abb. 7 Änderung der mittleren Niederschlagsmenge bei Auftreten einer Vb-Zugbahn im Sommerhalbjahr (Mai-Oktober) aus den Globalen Klimamodellen Echam5 (Quadrat), Echam6 (Kreis) und EC-Earth (Dreieck) für den Zeitraum 2071-2100 im Vergleich zu 1971-2000. Rosa:RCP4.5; Rot:SRES_A1B;Braun:RCP8.5 Klimaszenario. Die Zugbahnen wurden aus der Luftdruckverteilung auf $700 \mathrm{hPa}$ und Meeresniveau (SLP) abgeleitet

zeigen, dass konvektive Sommerniederschläge in den Alpen ergiebiger werden bei gleichbleibender Häufigkeit, die Häufigkeit großräumiger, skaliger Niederschläge jedoch stark abnimmt (Giorgi et al. 2016). In den Simulationen wird also das skalige Niederschlagsdefizit durch konvektiven Niederschlag kompensiert. Generell kann man aus den Simulationen mit regionalen Klimamodellen ableiten, dass die Intensität der Starkniederschläge auf Stundenund Tagesbasis im Sommerhalbjahr um $+7 \%$ pro ${ }^{\circ} \mathrm{C}$ Zunahme der Lufttemperatur ansteigen werden (weiche Aussage) (Westra et al. 2014; Frei et al. 2006; Trenberth et al. 2003).

Die Vorgängerstudie definierte Mechanismen möglicher Veränderungen von Hochwässern in Österreich (Blöschl et al. 2011b): Zunahme des Winterniederschlags, Abnahme des Sommerniederschlags (z.B. durch Verschiebung der Zirkulationsmuster) (mittelharte Aussage); Zunahme der Lufttempera-

Tab. 2 Möglicher prozentueller Einfluss eines veränderten Klimas auf das $\mathrm{HQ}_{100}$ in einem typischen Gebiet nördlich und südlich des Alpenhauptkammes in Österreich. 2021-2050 im Vergleich zu 1976-2007. Ergebnis der Monte-CarloSensitivitätsanalysen aus der Vorgängerstudie und Aktualisierung tur, dadurch Erhöhung des Anteiles konvektiven Niederschlags und dadurch höhere Niederschlagsintensitäten (weiche Aussage); Zunahme der Lufttemperatur, dadurch Erhöhung der Schneefallgrenze, dadurch größerer Anteil flüssigen Niederschlags von Ereignissen (harte Aussage); Zunahme der Lufttemperatur, dadurch Verschiebung der Schneeschmelze in das Frühjahr, dadurch Verschiebung des Jahresverlaufes des Abflussbeiwertes; Erhöhung der Verdunstung, dadurch Verringerung des Abflussbeiwertes (harte Aussage). Für jeden Mechanismus wurden in der Vorgängerstudie Monte-Carlo-Simulationen für den Zeitraum 2021-2050 im Vergleich zu 1976-2007 durchgeführt. Für zehn hydroklimatologische Regionen in Österreich bestanden die Simulationen aus drei Schritten: (a) Erzeugen von Niederschlagszeitreihen mittels eines stochastischen Niederschlagsmodells; (b) Umsetzen dieser Zeitreihen in Abflusszeitreihen mittels eines Nie-
derschlag-Abflussmodells; (c) Analyse der Eigenschaften der Niederschlagsund Abflussereignisse. Die Parameter der Modelle wurden für die Szenarien des Zeitraums 2021-2050 im Vergleich zu 1976-2007 verändert. Beispielsweise wurden für den ersten Mechanismus die Parameter des stochastischen Niederschlagsmodells so verändert, das die Winter und Sommerniederschläge den Projektionen der Klimamodelle entsprechen. Im Vergleich zu Vorgängerstudie sind nun die folgenden neuen Erkenntnisse verfügbar:

- Während das in der Vorgängerstudie verwendete Regionale Klimamodell COSMO-CLM eine deutliche Abnahme der Sommerniederschläge im Westen Österreichs prognostizierte, ergeben die neuen Modellläufe aus EURO-CORDEX keine Abnahme. In der Vorgängerstudie wurde in den Monte-Carlo-Simulationen entsprechend eine Abnahme von -4 bis $-7 \%$ (2021-2050 im Vergleich zu 1976-2007) angesetzt. Nach den neuen Simulationen sind es 0 bis $+5 \%$ (mittelharte Aussage) (Abb. 4).

- In der Vorgängerstudie wurde angenommen, dass eine Zunahme der konvektiven Niederschlagsmengen mit zunehmender Temperatur wahrscheinlich ist. Die neuen Auswertungen von Wang et al. (2017), Lepore et al. (2015) und Utsumi et al. (2011) deuten jedoch darauf hin, dass der Effekt der Temperaturzunahme auf konvektive Niederschläge in Österreich geringer als zuvor angenommen ist. Die Parameter des stochastischen Niederschlagmodells für die Monte-Carlo-Simulationen wurden in der alten Studie so gewählt, dass die Zunahme des 100-jährlichen Niederschlags für eine Dauer von $6 \mathrm{~h}$ je nach Region zwischen 2 und $18 \%$ betrug (im Mittel $7 \%$ ). Nach dem aktuellen Informationsstand dürfen etwas kleinere Werte realistischer sein (weiche Aussage).

Auf Basis dieser neuen Erkenntnisse wurde nun abgeschätzt, wie die Ergeb- 


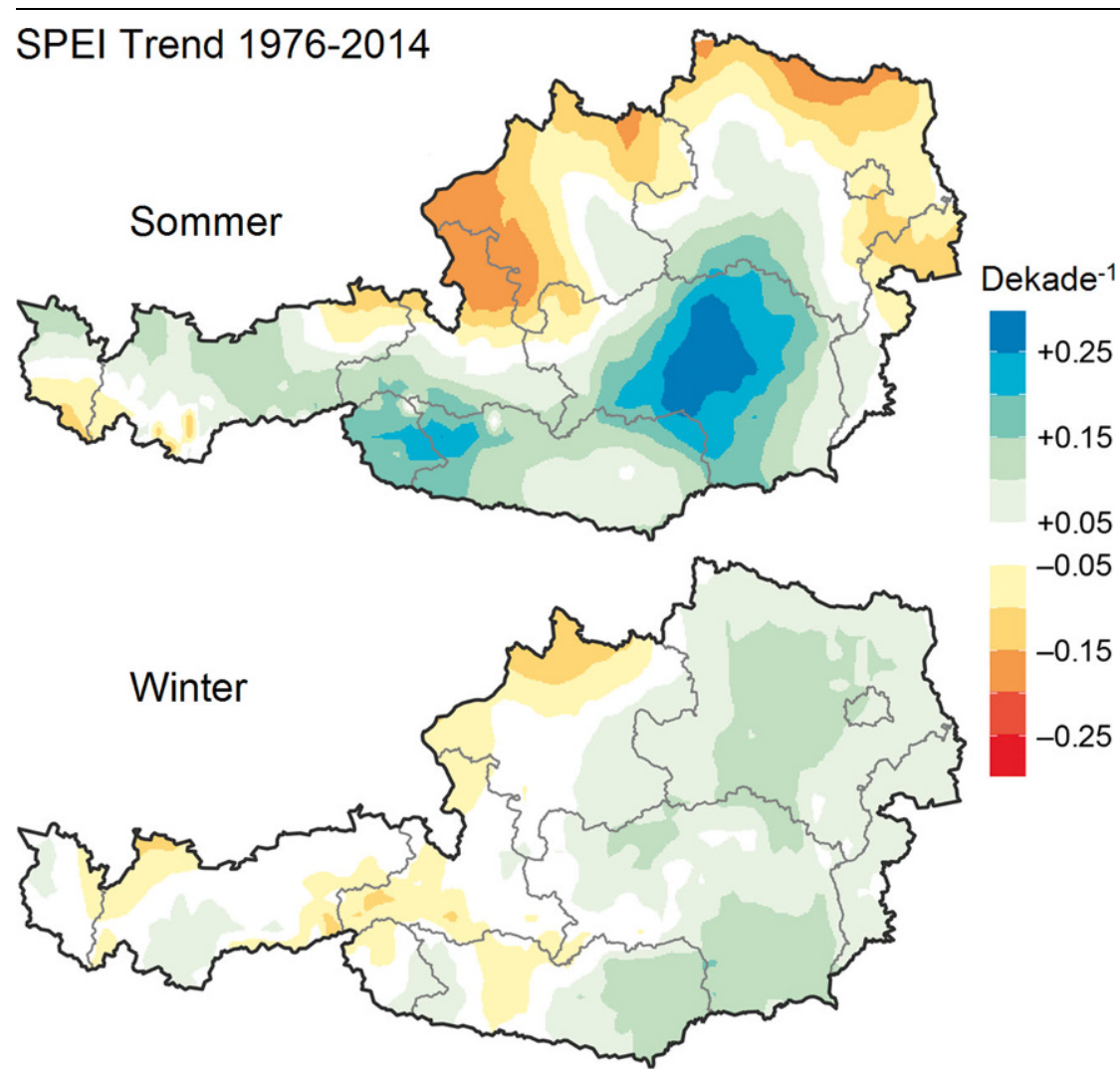

Abb. 8 Trends des Standardized Precipitation Evapotranspiration Index (SPEI) im Sommer (Juni-August) und Winter (Dezember-Februar; Laaha et al. 2014). Ein Trend von-0,1 pro Dekade entspräche einer Änderung von im Mittel normalen Bedingungen (SPEI 0) hin zu im Mittel mäßig trockenen Bedingungen (SPEI -1) über einen Zeitraum von 100 Jahren (vgl. Vincente-Serrano et al. 2010; McKee et al. 1993)

nisse der Monte-Carlo-Simulationen anzupassen sind (Tab. 2). Im Vergleich zur Vorgängerstudie verschiebt sich in Westösterreich eine Abnahme der $\mathrm{HQ}_{100}$ um bis $\mathrm{zu}-4 \%$ auf nahezu keine Änderung. In Ost- und Südösterreich verschiebt sich eine Zunahme der $\mathrm{HQ}_{100} \mathrm{um}$ bis $\mathrm{zu}+7 \%$ auf eine geringere Zunahme von bis $\mathrm{zu}+5 \%$. In den anderen Regionen (Traun, Enns, Erlauf, Traisen, Innviertel, Mühlviertel, Waldviertel) gibt es keine Änderung im Vergleich zur Vorgängerstudie. Insbesondere in der Region Innviertel, Mühlviertel bleibt die erwartete Zunahme von $+10 \%$ bestehen. Im Mittel über alle Regionen war die Änderung des $\mathrm{HQ}_{100}$ in der Vorgängerstudie $+2 \%$, durch die Aktualisierung ändert sich dieser Wert nicht. Das bedeutet, dass im Wesentlichen die Aussagen der Vorgängerstudie durch die neuen Informationen bestätigt werden.

Ein alternativer Ansatz zur Bestimmung sich veränderter Hochwasserwahrscheinlichkeiten ist die instationäre Hochwasserstatistik (Delgado et al.
(Vergangenheit) zeigt folgendes Bild: Im Innviertel und Mühlviertel ergeben sowohl die Trendanalysen als auch die Szenarien Anstiege. Am nördlichen Alpenrand zeigen die Trendanalysen Anstiege (möglicherweise wegen veränderter atmosphärischer Zirkulationsmuster), die Szenarien zeigen jedoch etwa gleichbleibende Hochwässer wegen geringer Änderung in den projizierten Monatsniederschlägen und der früheren Schneeschmelze. Die Aussage, dass die natürliche Variabilität der Hochwässer wesentlich größer ist als die Änderung zufolge des Klimawandels, bleibt bestehen.

\section{Niederwasser und Grundwasserneubildung}

\subsection{Situation in der Vergangenheit}

Zur Beschreibung trockener meteorologischer Bedingungen und deren Trends können Dürre-Indizes wie der Standardized Precipitation Evapotranspiration Index (SPEI) herangezogen werden, der die klimatische Wasserbilanz (Niederschlag minus potenzieller Verdunstung) abbildet (Vincente-Serrano et al. 2010). Trends des SPEI für den Zeitraum 1976-2014 auf Basis der HISTALP Daten (Auer et al. 2007; Haslinger et al. 2014) (Abb. 8) ähneln im Winter den Trends des Niederschlags. Im Sommer sind in manchen Gebieten Österreichs (Norden, Osten) negative Trends des SPEI festzustellen, obwohl der Niederschlag in diesem Zeitraum leicht zugenommen hat. Dies ist auf den Anstieg der potenziellen Verdunstung zurückzuführen. Duethmann und Blöschl (2018) zeigten durch einen Vergleich mehrerer Methoden (z.B. Penman-Monteith, Verdunstungswannen), dass die potenzielle Verdunstung vor allem in den 1980er- und 1990er-Jahren wegern höherer Lufttemperatur und stärkerer Einstrahlung stark zugenommen hat (siehe auch Abb. 3 und Brunetti et al. 2009).

Trendauswertungen der beobachteten Jahresniederwasserdurchflüsse Q95 (der Durchfluss der im jeweiligen Jahr an $95 \%$ der Tage überschritten wird) in Österreich für den Zeitraum 1976-2014 (Abb. 9, Tab. 3, harte Aussagen) zeigen ähnliche Änderungen wie im Zeitraum 1976-2007. Im Alpenraum sind größtenteils Zunahmen der Niederwasserdurchflüsse $\mathrm{zu}$ verzeichnen. In Gebieten mit einer Seehöhe von über $900 \mathrm{~m}$ zeigen $22 \%$ der Pegel- 


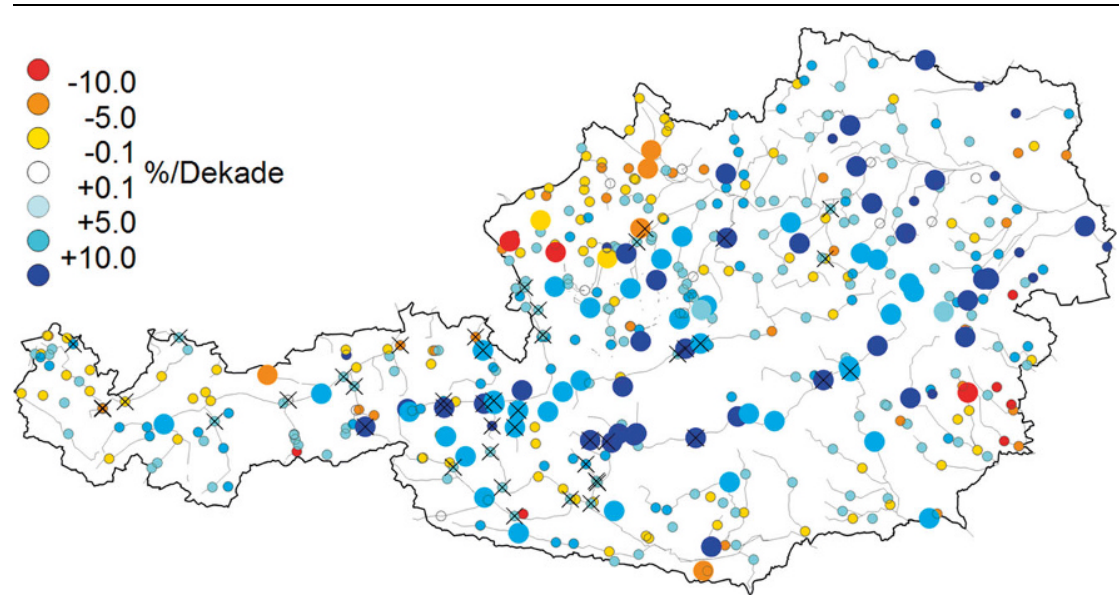

Abb. 9 Trends der Jahresniederwasserdurchflüsse Q95 für den Zeitraum 1976-2014. Große Kreise blau: signifikant steigende Trends, d. h. Zunahme des Q95. Große Kreise rot: signifikant fallende Trends. Kleine Kreise: Trends nicht signifikant (Signifikanzniveau $5 \%$ ). Kreuze zeigen Pegel, die durch Überleitungen oder Speicher beeinflusst sind

einzugsgebiete einen signifikant steigenden Trend (Vorgängerstudie $12 \%$ ). Dem gegenüber haben nur in $1 \%$ (Vorgängerstudie $3 \%$ ) der Gebiete die Niederwasserdurchflüsse signifikant abgenommen. Dies ist auf den geringeren Schneerückhalt (Abb. 2) zurückzuführen. Ein größerer Teil des Niederschlags kommt als Regen direkt zum Abfluss und die Schneedeckenperiode ist kürzer, wodurch sich die Durchflüsse im Winterhalbjahr, in denen die Niederwässer auftreten, erhöhen.

Unter $900 \mathrm{~m}$ Seehöhe haben in $4 \%$ der Gebiete die Niederwasserdurchflüsse signifikant abgenommen, vor allem im Innviertel. Die Abnahme der Durchflüsse fällt deutlich geringer aus als in der Vorgängerstudie, in der $10 \%$ der Gebiete signifikant abnehmende Niederwasserdurchflüsse zeigten. Im Flachland des Nordens, Ostens sowie Südostens besteht der wesentliche Niederwassermechanismus in der Verdunstung im Sommer, die gestiegen ist. Obwohl in den letzten Jahrzehn- ten auch die Sommerniederschläge gestiegen sind (Abb. 1), führte die stärkere Verdunstung zu (nicht signifikant) fallenden Trends des Niederwasserdurchflusses an mehreren Pegeln. Der mittlere Trend der Q95 in Gebieten über $900 \mathrm{~m}$ betrug $+3,7 \% /$ Dekade (Vorgängerstudie $+2,3 \%$ /Dekade), in Gebieten unter $900 \mathrm{~m}$ betrug er $+2,2 \% /$ Dekade

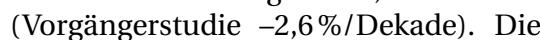
abnehmenden Trends im Flachland haben sich also im Vergleich zur Vorgängerstudie abgeschwächt. Das ist darauf zurückzuführen, dass die letzten Jahre eher niederschlagsreich waren.

Eine Analyse der einzelnen Wasserbilanzkomponenten erlaubt auch Aufschlüsse über klimainduzierte Veränderungen hinsichtlich der Grundwasserneubildung und damit auch hinsichtlich der quantitativen Grundwasserverhältnisse in Form der Grundwasserstände. Während der Jahresniederschlag im letzten Jahrzehnt auf einem relativ hohen Niveau im Vergleich zum Mittelwert des 20. Jahrhunderts lag, war die jahreszeitliche Verteilung unterschiedlich. Die Sommerniederschläge haben deutlich zugenommen (+14\% für den Zeitraum 1996-2014 im Vergleich zu 1976-1995), die Winterniederschläge hingegen abgenommen (-7\%) (Abb. 1). Die Winter 2008-2014 waren im Süden und Südosten Österreichs niederschlagsreich. Eine Erhöhung des Wintersniederschlags lässt auf eine erhöhte Grundwasserneubildung im Frühjahr schließen. Entsprechend der Entspannung der Niederwassertrends dürften sich die abnehmenden Trends der Grundwasserneubildung in den letzten Jahren abgeschwächt haben (mittelharte Aussage). Im Westen waren die Winter 2008-2014 eher niederschlagsarm, was auf eine etwas reduzierte Grundwasserneubildung schließen lässt. Trendanalysen im Zeitraum 1976-2006 (Blaschke et al. 2011) zeigten, dass rund $70 \%$ der Messstellen in Österreich keinen signifikanten, $12 \%$ einen signifikant steigenden und $18 \%$ einen signifikant fallenden Trend im Jahresmittel des Grundwasserstandes aufweisen. Das heterogene Bild aus der Vorgängerstudie dürfte auch mit den aktualisierten Informationen zur Wasserbilanz Gültigkeit haben. Diese Interpretation wird durch die verhältnismäßig gleichbleibenden Jahresabflüsse im Mittel über Österreich (Abb. 3) gestützt.

\subsection{Situation in der Zukunft}

Haslinger et al. (2015) wertete ein Ensemble an regionalen Klimasimulationen mit unterschiedlichen Treibhausgasszenarien für den Alpenraum aus, um zukünftige Dürresituationen abzuschätzen. Die Modelle zeigen gegen Ende des 21. Jahrhunderts meist deutlich trockenere Bedingungen gemessen am SPEI als derzeit. Diese Änderung geht auch mit einer Erhöhung der Varianz zwischen den Jahren einher. Die Ände-

Tab. 3 Prozent der Pegel mit signifikant steigenden und fallenden Trends der Q95 Niederwasserdurchflüsse in Österreich. $h$ ist die mittlere Einzugsgebietshöhe. Entsprechend dem gewählten Signifikanzniveau von $5 \%$ (jeweils $5 \%$ an den beiden Enden der Verteilung) sind Werte von $5 \%$ und kleiner als zufällig anzusehen

\begin{tabular}{|c|c|c|c|c|c|c|c|}
\hline & \multirow[t]{3}{*}{ Anzahl der Pegel } & \multicolumn{3}{|c|}{$\begin{array}{l}\text { 1976-2007 } \\
\text { (min. 25 Jahre) }\end{array}$} & \multicolumn{3}{|c|}{$\begin{array}{l}\text { 1976-2014 } \\
\text { (min. 32 Jahre) }\end{array}$} \\
\hline & & \multicolumn{3}{|c|}{ Anteil d. Pegel [\%] mit } & \multicolumn{3}{|c|}{ Anteil d. Pegel [\%] mit } \\
\hline & & $\begin{array}{l}\text { Steigendem } \\
\text { Trend }\end{array}$ & $\begin{array}{l}\text { Nicht signif. } \\
\text { Trend }\end{array}$ & $\begin{array}{l}\text { Fallendem } \\
\text { Trend }\end{array}$ & $\begin{array}{l}\text { Steigendem } \\
\text { Trend }\end{array}$ & $\begin{array}{l}\text { Nicht signif. } \\
\text { Trend }\end{array}$ & $\begin{array}{l}\text { Fallendem } \\
\text { Trend }\end{array}$ \\
\hline Alle Gebiete in Österreich & 458 & 7 & 86 & 7 & 16 & 82 & 2 \\
\hline $\begin{array}{l}\text { Seehöhe } \\
h<900 \mathrm{~m}\end{array}$ & 222 & 3 & 87 & 10 & 11 & 85 & 4 \\
\hline $\begin{array}{l}\text { Seehöhe } \\
h>900 \mathrm{~m}\end{array}$ & 236 & 12 & 85 & 3 & 22 & 77 & 1 \\
\hline
\end{tabular}




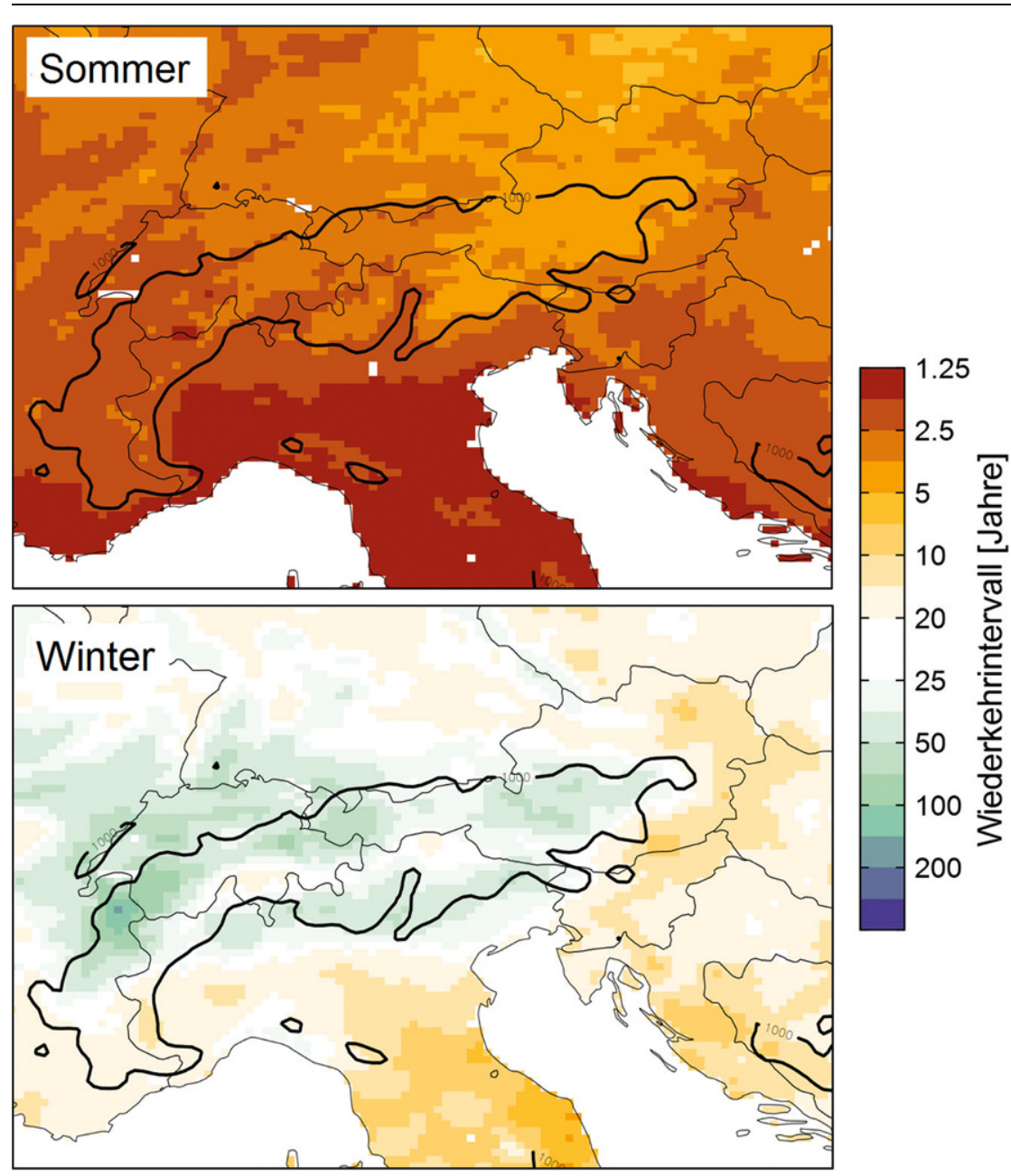

Abb. 10 Wiederkehrintervall eines extremen Dürrezustands (SPEI $<-2)$ im Sommer (Juni-August) und Winter (Dezember-Februar) für den Zeitraum 207-2100 im Vergleich zu 1971-2000 (Wiederkehrintervall 23 Jahre); braune Farben zeigen eine höhere Wahrscheinlichkeit der Dürre an. Globales Klimamodell ECHAM5, regionales Klimamodell COSMO-CLM, Szenario A1B (Haslinger et al. 2015)

rung des Wiederkehrintervalls für ein extremes Dürre-Ereignis, welche für die Referenzperiode (1971-2000) bei 23 Jahren liegt, ist in Abb. 10 für die Winter und Sommer im Zeitraum 2071-2100 dargestellt. Im Winter werden in den alpinen Gebieten die Wahrscheinlichkeiten der Dürre kleiner, im Flachland zeigen sich nur geringe Änderungen. Im Gegensatz dazu ist im Sommer eine stark erhöhte Wahrscheinlichkeit für Dürre zu verzeichnen, wobei dieses $\mathrm{Si}$ gnal südlich der Alpen noch deutlicher ist als im Norden.

Seit der Vorgängerstudie wurden verschiedene Simulationsrechnungen für zukünftige Niederwässer und Dürren in Österreich durchgeführt (siehe z. B. Laaha et al. 2016). Dabei wurden die Ergebnisse globaler Klimamodelle mittels regionaler Klimamodelle hin- mulationen geben einen Hinweis auf die Unsicherheiten. Die Zunahme der Niederwasserdurchflüsse in den Alpen ist bei beiden Simulationsrechnungen eindeutig, die Abnahme im Osten und Südosten ist weniger klar. Da im Osten und Südosten der Abfluss besonders sensitiv auf das Niederschlagsverhalten reagiert (Blaschke et al. 2011) ist auch dessen Unsicherheit zufolge des Niederschlags groß. Ändern sich die Niederschläge nicht, ist für den Osten und Südosten zufolge der erhöhten Verdunstung mit einer deutlichen Abnahme der Niederwasserdurchflüsse $\mathrm{zu}$ rechnen. Im ungünstigsten Fall kann diese Abnahme bis $\mathrm{zu} 30 \%$ betragen (Abb. 11 unten).

Die Szenarienrechnungen lassen für den Zeitraum 2021-2050 gegenüber 1971-2000 eine Zunahme der Winterniederschläge in ganz Österreich erkennen (Abb. 4). Diese kann zu einer Zunahme der Grundwasserneubildung führen, die vorwiegend im Winter und im Frühjahr stattfindet. Andererseits wird ein Teil des zusätzlichen Niederschlags durch die erhöhte Verdunstung aufgebraucht, die besonders im Osten Österreichs groß ist. In den niederschlagsarmen Regionen im Osten Österreichs können damit eher sinkende bzw. möglicherweise sich wenig ändernde Grundwasserstände erwartet werden (mittelharte Aussage). Dies entspricht der Aussage der Vorgängerstudie. In den Alpen und nördlich der Alpen ist eher eine Zunahme der Grundwasserneubildung zu erwarten. Für den Süden Österreichs (Kärnten, Steiermark), wo nun eine Zunahme der Winterniederschläge erwartet wird, ist mit einer Zunahme oder einem Gleichbleiben der Grundwasserneubildung zu rechnen (mittelharte Aussage). Im Vergleich dazu hatte die Vorgängerstudie im Süden Österreichs einer Abnahme der Grundwasserneubildung angegeben. Prognosen über Änderungen der Grundwasserstände einzelner Messstellen sind jedoch nach dem derzeitigen Kenntnisstand nicht möglich, da diese von den lokalen Verhältnissen einschließlich der menschlichen Eingriffe geprägt werden.

\section{Zusammenfassung und Schlussfolgerungen}

Die Verdunstung hat in den letzten drei Jahrzehnten im Mittel für Österreich um etwa $80 \mathrm{~mm} / \mathrm{a}$ zugenommen, der Jahresniederschlag ebenfalls ent- 


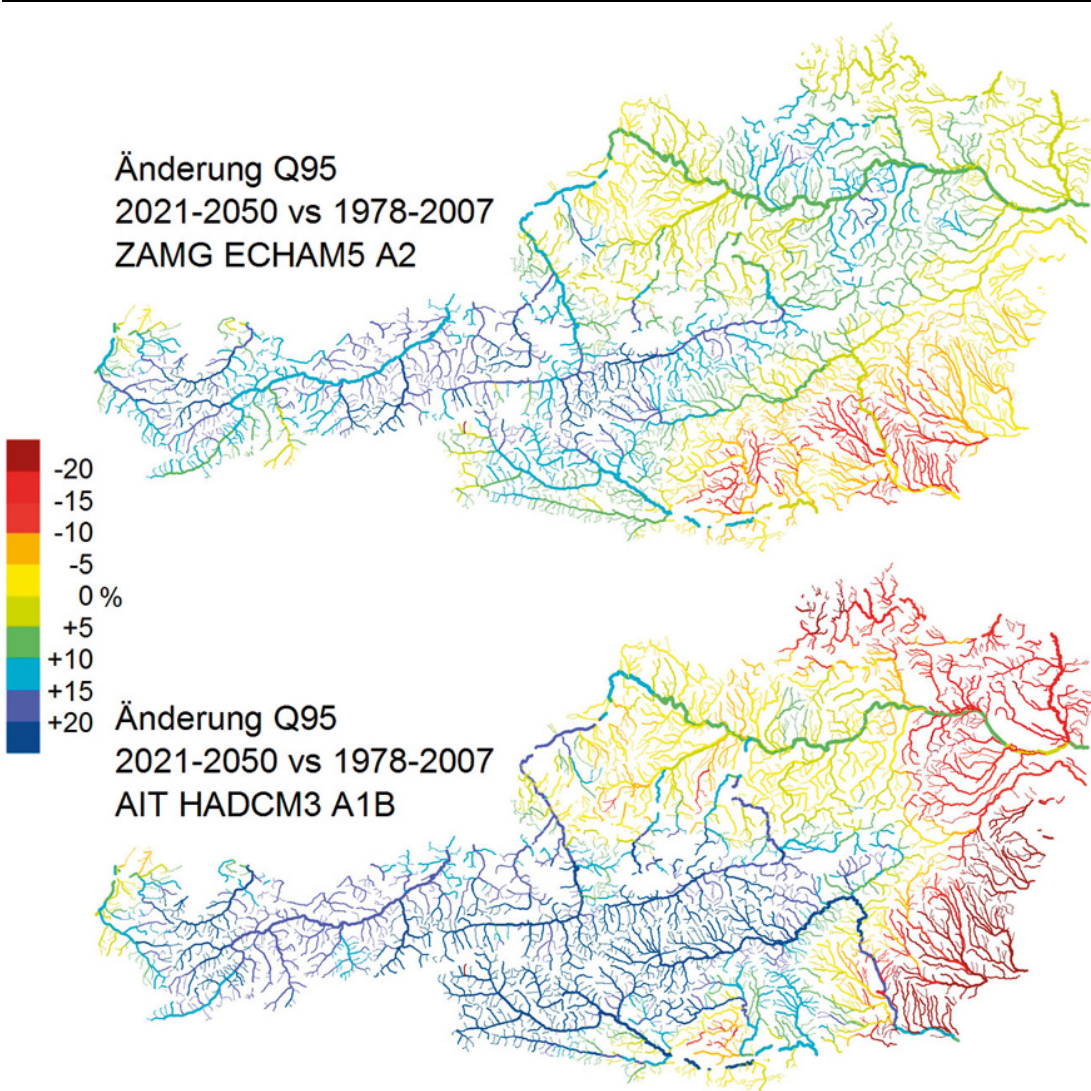

Abb. 11 Änderung des Q95-Niederwasserdurchflusses für den Zeitraum 2021-2050 im Vergleich zu 1978-2007 berechnet mit einem kontinuierlichen NiederschlagAbflussmodel. Oben: globales Modell ECHAM5, regionales Modell CLM ZAMG, Klimaszenario A2. Unten: globales Modell HadCM3, regionales Modell CLM AIT, Klimaszenario A1B. Blau: Zunahme des Q95, rot: Abnahme des Q95 (Laaha et al. 2014)

sprechend zugenommen, wodurch sich der Abfluss wenig geändert hat (harte Aussage). Für die Zukunft (Zeithorizont 2021-2050 im Vergleich zur derzeitigen Situation) könnte im Südosten Österreichs der mittlere jährliche Abfluss abnehmen (weiche Aussage). Die natürliche Variabilität des mittleren jährlichen Abflusses zwischen den Jahren ist größer als die zufolge Klimaänderung im Zeitraum 2021-2050 zu erwartenden Änderungen (mittelharte Aussage).

Im Vergleich zur Vorgängerstudie haben sich die zunehmenden Trends der gemessenen Hochwasserabflüsse etwas verstärkt. Dadurch hat sich die Hochwasserproblematik ebenfalls verschärft ( harte Aussage). Starkniederschlagsrelevante Zugbahnen dürften in den nächsten Jahrzehnten an Häufigkeit nicht zunehmen, aber könnten intensivere Niederschläge bringen. Für die Zukunft (Zeithorizont 2021-2050 im Vergleich zur derzeitigen Situation) werden regional unterschiedliche Änderungen der Abflüsse bei Hochwässern $\left(\mathrm{HQ}_{100}\right)$ er-
Niederwasser von etwa 10-20\% eintreten (mittelharte Aussage). Eine Orientierung über die wasserwirtschaftlichen Konsequenzen der Niederwassersituationen bei Klimaänderung im Sommer können die Niederwasserjahre 2003 und 2015 geben.

Entsprechend der Entspannung der Niederwassertrends dürften sich die abnehmenden Trends der Grundwasserneubildung in den letzten Jahren abgeschwächt haben (mittelharte Aussage). Für die Zukunft (Zeithorizont 2021-2050 im Vergleich zur derzeitigen Situation) wird ein regional unterschiedliches Verhalten erwartet. Im Süden Österreichs, wo eine Zunahme der Winterniederschläge erwartet wird, könnte die Grundwasserneubildung zunehmen oder gleich bleiben. Im Norden und Westen Österreichs könnte sie zunehmen, und in den niederschlagsarmen Regionen im Osten Österreichs ist künftig eine Abnahme der Grundwasserneubildung oder eine geringere Änderung wahrscheinlich (mittelharte Aussage).

Insgesamt sind die Änderungen im Vergleich zur Vorgängerstudie (ZAMG und TU Wien 2011) nicht sehr groß. Damit sind auch die im Nationaler Gewässerbewirtschaftungsplan 2015 (BMLFUW 2015) dargestellten Anpassungsstrategien weiterhin aktuell. Die zusätzlich empfohlenen Anpassungsmaßnahmen beschränken sich deshalb auf wenige Punkte, wie etwa, Oberflächenabfluss und Hangwasser (pluviales Hochwasser) verstärkte Aufmerksamkeit zu widmen. Weiterhin sind die etwas zunehmenden Hochwässer im Norden Österreichs im Auge zu behalten, sowie die zunehmende Verdunstung, die sich negativ auf die Wasserverfügbarkeit auswirken könnte.

Danksagung Diese Studie wurde durchgeführt im Auftrag des BMNT, Sektion I $\mathrm{V}$ und der wasserwirtschaftlichen Abteilungen der Ämter der Landesregierungen Österreichs. Wir danken den Mitarbeiterinnen und Mitarbeitern dieser Institutionen für die konstruktive Begleitung der Studie. Die Daten wurden vom Hydrographischen Zentralbüro, den hydrografischen Diensten der Länder und der Zentralanstalt für Meteorologie und Geodynamik zur Verfügung gestellt.

Funding Open access funding provided by TU Wien (TUW). 
Open Access Dieser Artikel wird unter der Creative Commons Namensnennung 4.0 International Lizenz (http:// creativecommons.org/licenses/by/4. $0 /$ deed.de) veröffentlicht, welche die
Nutzung, Vervielfältigung, Bearbeitung, Verbreitung und Wiedergabe in jeglichem Medium und Format erlaubt, sofern Sie den/die ursprünglichen $\mathrm{Au}$ tor(en) und die Quelle ordnungsgemäß nennen, einen Link zur Creative Commons Lizenz beifügen und angeben, ob Änderungen vorgenommen wurden.
Alaoui, A., Rogger, M., Peth, S. and Blöschl, G. (2018): Does soil compaction increase floods? A review. Journal of Hydrology, 557, 631-642, https://doi.org/10.1016/j.jhydrol.2017.12.052.

Auer, I., Böhm, R., Jurkovic, A., Lipa, W., Orlik, A., Potzmann, R., Schöner, W., Ungersböck, M. Matulla, C., Briffa, K., Jones, P. D., Efthymiadis, D., Brunetti, M, Nanni. T., Maugeri, M., Mercalli, L., Mestre, O., Moisselin, J.-M., Begert, M. Müller-Westermeier, G., Kveton, V., Bochnicek O., Stastny, P., Lapin, M., Szalai, S., Szentimrey, T., Cegnar, T., Dolinar, M., Gajic-Capka, M., Zaninovic, K., Majstorovic, Z., Nieplova, E. (2007): HISTALP - Historical instrumental climatological surface time series of the greater Alpine region 1760-2003. International Journal of Climatology, 27, 17-46.

Blaschke, A. P., Merz, R., Parajka, J., Salinas, J. und Blöschl, G. (2011): Auswirkungen des Klimawandels auf das Wasserdargebot von Grund und Oberflächenwasser. Österreichische Wasserund Abfallwirtschaft, 63, (1-2), 31-41.

Blöschl, G. et al. (2017b): Changing climate shifts timing of European floods. Science, 357 (6351) 588-590, doi: 10.1126/science.aan2506

Blöschl, G., Viglione, A., Merz., R., J. Parajka, J. Salinas, J. und Schöner, W. (2011b): Auswirkungen des Klimawandels auf Hochwasse und Niederwasser. Österreichische Wasser- und Abfallwirtschaft, 63, (1-2), 21-30.

Blöschl, G., Schöner, W., Kroiß, H., Blaschke, A. P., Böhm, R., Haslinger, K., Kreuzinger, N. Merz, R., Parajka, J., Salinas, J. L., Viglione, A (2011a): Anpassungsstrategien an den Klimawandel für Österreichs Wasserwirtschaft - Ziele und Schlussfolgerungen der Studie für Bund und Länder. Österreichische Wasser- und Abfallwirtschaft, 63, (1-2), 1-10.

Blöschl, G., Nester, Th., Komma, J., Parajka J. and Perdigão R.A.P. (2013): Das Juni-Hochwasser 2013 - Analyse und Konsequenzen für das Hochwasserrisikomanagement. Österreichische Ingenieur- und Architekten-Zeitschrift, 158, 141-152.

Blöschl, G., Parajka, J., Blaschke, A. P., Hofstätter, M., Haslinger, K. und Schöner, W. (2017a): Klimawandel in der Wasserwirtschaft - Schwerpunkt Hochwasser, Dürre und Trockenheit Bundesministerium für Land- und Forstwirtschaft, Umwelt und Wasserwirtschaft. https:// www.bmnt.gv.at/wasser/wasser-oesterreich/ foerderungen/trinkwasser_abwasser/aktuelle projekte/klimawandel_wasserwirtschaft.html. Zugegriffen: 20. Juni 2018

Blöschl, G., Komma, J., Nester, T., Rogger, M. Salinas, J. und Viglione, A. (2018): Die Wirkung des Waldes auf Hochwässer. Wildbach- und Lawinenverbau, 88, (181), 288-296.

BMLFUW (2015): Nationaler Gewässerbewirtschaftungsplan 2015. Bundesministerium für Land- und Forstwirtschaft, Umwelt und Wasserwirtschaft, Wien.

Brunetti, M., Lentini, G., Maugeri, M., Nanni, T., Auer, I., Böhm, R., Schöner W. (2009): Climate variability and change in the Greater Alpine Region over the last two centuries based on multi- variable analysis. Int. J. Climatol., 29, 2197-2225, https://doi.org/10.1002/joc.1857.

Chimani, B., Heinrich, G., Hofstätter, M. Kerschbaumer, M., Kienberger, S., Leuprecht, A., Lexer, A., Peßenteiner, S., Poetsch, M. S. Salzmann, M., Spiekermann, R., Switanek, M. und Truhetz, H. (2016): ÖKS15 - Klimaszenarien für Österreich. Daten, Methoden und Klimaanalyse. Projektendbericht, Zentralanstalt für Meteorologie und Geodynamik, Wien. ISBN 978-3-903171-02-2. https://hdl.handle.net/20. $500.11756 / 06$ edd0c9

Delgado, J. M., Merz, B., Apel, H. (2014): Projecting flood hazard under climate change: an alternative approach to model chains. Natural $\mathrm{Ha}$ zards and Earth System Science, 14, 6, 1579-1589. Duethmann, D. and Blöschl, G. (2018): Why has catchment evaporation increased in the past 40 years? A data-based study in Austria. Hydrol. Earth Syst. Sci. Discuss., https://doi.org/10.5194/ hess-2018-129

Frei, C., Schöll, R., Fukutome, S., Schmidli, J. and Vidale, P. L. (2006), Future change of precipitation extremes in Europe: Intercomparison of scenarios from regional climate models, $J$. Geophys. Res., 111, D06105, https://doi.org/10. 1029/2005JD005965.

Giorgi, F., Torma, Cs., Coppola, E., Ban, N. Schär, C., Somot, S. (2016): Enhanced summe convective rain at Alpine high elevations in response to climate warming. Nature Geoscience, 9, 584-589. https://doi.org/10.1038/ngeo2761.

Hall, J., Arheimer, B., Borga, M., Brázdil, R. Claps, P., Kiss, A., Kjeldsen, T. R., Kriaučiūnienè, J., Kundzewicz, Z. W., Lang, M., Llasat, M. C., Macdonald, N., McIntyre, N., Mediero, L., Merz, B., Merz, R., Molnar, P., Montanari, A., Neuhold, C., Parajka, J., Perdigão, R. A. P., Plavcová, L., Rogger, M., Salinas, J. L., Sauquet, E., Schär, C., Szolgay, J., Viglione, A. and Blöschl, G. (2014): Understanding Flood Regime Changes in Europe: A state of the art assessment. Hydrology and Earth System Sciences, 18, 2735-2772, https:// doi.org/10.5194/hess-18-2735-2014.

Haslinger, K., Koffler, D., Schöner, W. and Laaha, G. (2014): Exploring the link between me teorological drought and streamflow: Effects of climate-catchment interaction, Water Resour Res., 50, 2468-2487, https://doi.org/10.1002/ 2013WR015051

Haslinger, K., Schöner, W. and Anders, I. (2015): Future drought probabilities in the Greater Alpine Region based on COSMO-CLM experiments Spatial patterns and driving forces, Meteorologische Zeitrschrift, 25 (2), https://doi.org/10.1127/ metz/2015/0604.

Hiebl, J. and Frei, C. (2016): Daily temperature grids for Austria since 1961 - concept, creation and applicability. Theoretical and Applied Climatology, 124 (1-2), 161-178.

Hofstätter, M., Jacobeit, J., Homann, M., Lexer, A., Chimani, B., Philipp, A., Beck, C. und Ganekind, M. (2015): WETRAX - Weather Patterns, Cyclone Tracks and related Precipitation Extremes. Großflächige Starkniederschläge im Klimawandel in Mitteleuropa. Projektendbericht.
Geographica Augustana 19. ISBN: 3-923273-966; ISSN: 1862-8680;

Hofstätter, M., Lexer, A., Homann, M. and Blöschl, G. (2017): Large-scale heavy precipitation over central Europe and the role of atmospheric cyclone track types. Int J Climatol. https://doi.org/10.1002/joc.5386.

Laaha, G., Blöschl G. and Schöner, W. (2014): Climate Impact on Low Flows And Droughts, Endbericht, ACRP2, https://www.klimafonds.gv. at/assets/Uploads/Projektberichte/ACRP-2009/ 20150716CILFADEBACRP2B060362.pdf. Laaha, G., Haslinger, K., Koffler, D., Parajka J., Schöner, W., Viglione, A., Zehetgruber, J., Blöschl G. (2016): Ein Drei-Standbeine-Ansatz zur Ermittlung zukünftiger Niederwasserabflüsse in Österreich. Österreichische Wasser- und Abfallwirtschaft, 68, 54-57, doi 10.1007/s00506015-0284-1

Lepore, C., Veneziano, D. and Molini, A. (2015): Temperature and CAPE dependence of rainfall extremes in the eastern United States, Geophys. Res. Lett., 42, 74-83, https://doi.org/10.1002/ 2014 GL062247.

Lexer, A., Höfler, A., Hofstätter, M., Krennert, T. (2018): CONVEX: Konvektive Starkniederschläge in Österreich von 1986-2015. Endbericht, Zentralanstalt für Meteorologie und Geodynamik, Wien.

McKee, T. B., Doeskin, N. J. and Kleis, J. (1993): The relationship of drought frequency and duration to time scales. Preprints, 8th Conf. on Applied Climatology, Anaheim, CA, Amer. Meteor. Soc., 179-184.

Minder, J. R. (2010): On the Climatology of Orographic Precipitation in the Mid-Latitudes, Dissertation University of Washington, $187 \mathrm{pp}$.

Montanari, A., Blöschl, G., Sivapalan, M., Savenije, H. (2010): Getting on target. Public Service Review: Science and Technology, 7, 167-169. Perdigão, R. A. P., and Blöschl, G. (2014): Spatiotemporal flood sensitivity to annual precipitation: Evidence for landscape-climate coevolution, Water Resour. Res., 50, 5492-5509, https:// doi.org/10.1002/2014WR015365.

Schöner, W., Koch, R., Reisenhofer, S., Strasser, U., Marke, T., Marty, C., Tilg, A. (2016): Snow in Austria during the instrumental period - spatiotemporal patterns and their causes-relevance for future snow scenarios, Endbericht ACRP Projekt Snowpat, 43 pp..

Šraj , M., Viglione, A., Parajka, J. and Blöschl, G. (2016): The influence of non-stationarity in extreme hydrological events on flood frequency estimation. Journal of Hydrology and Hydromechanics, 64 (4), 426-437. https://doi.org/10. 1515/johh-2016-0032.

Trenberth, K. E., Dai, A., Rasmussen, R. M. and Parsons, D. B. (2003): The changing character of precipitation, Bull. Am. Meterol. Soc., 84, 1205-1217.

Utsumi, N., Seto, S., Kanae, S., Maeda, E. E. and Oki, T. (2011): Does higher surface temperature intensify extreme precipitation?, Geophys. Res. Lett., 38, L16708, https://doi.org/10.1029/ 2011GL048426. 
Viglione, A., Merz, B., Viet Dung, N., Parajka, J., Nester, T. and Blöschl, G. (2016): Attribution of regional flood changes based on scaling fingerprints. Water Resources Research, 52, 5322-5340, https://doi.org/10.1002/2016WR019036.

Vincente-Serrano, S. M., Beguería, S., and López-Moreno, J. I. (2010): A Multiscalar Drought Index Sensitive to Global Warming: The Standardized Precipitation Evapotranspiration Index,
J. Climate, 23, 1696-1718, https://doi.org/10. 1175/2009JCLI2909.1.

Wang, G., Wang, D., Trenberth, K. E., Erfanian, A., Yu, M., Bosilovich, M. G. and Parr, D. T.

(2017): The peak structure and future changes of the relationships between extreme precipitation and temperature. Nature Climate Change, 7 (4), 268-274.

Westra, S., Fowler, H. J., Evans, J. P., Alexander,

L. V., Berg, P., Johnson, F., Kendon, E. J., Lende- rink, G. and Roberts, N. M. (2014): Future changes to the intensity and frequency of shortduration extreme rainfall, Rev. Geophys., 52, 522-555, https:// doi.org/10.1002/2014RG000464.

ZAMG/TU Wien (2011): Anpassungsstrategien an den Klimawandel für Österreichs Wasserwirtschaft. Endbericht. Lebensministerium. Download https://www.bmlfuw.gv.at/wasser/wasseroesterreich/herausforderungen/klimawasser. html. Zugegriffen: 20. Juni 2018 